\title{
RAFIK SCHAMI - EIN ERZÄHLER ZWISCHEN ORIENT UND OKZIDENT Exemplarische Untersuchungen an den märchenhaften Erzählungen Als der Meister auftrat und Reise zwischen Nacht und Morgen ${ }^{1}$
}

\author{
Die Vergangenheit dehnt sich in die \\ Gegenwart hinein, drückt sie zu einer \\ bedeutungslosen dünnen Schicht \\ zusammen und schildert immer \\ farbiger und lebendiger als diese. \\ Wirklichkeit, Traum- und Wunschbilder \\ werden $[\ldots]$ vermischt $[\ldots]$, und diese \\ Synthese heißt vornehm: Lteratur. ${ }^{2}$
}

Noch nie war eine Sache weltweit so aktuell wie der Appell auf den Dialog der Kulturen. Die ersten Kontakte zwischen Morgenland und Abendland liegen schon weit in der Vergangenheit zurück. Doch im Unterschied zu früheren Zeiten genießen wir heute eine unheimlich schnelle Form von Kommunikation, die besonders durch die immer rasanter fortschreitende Technik gefördert wird und dementsprechend synchron-visuell die Grenzen zwischen den Ländern aufhebt und die ganze Welt miteinander verbindet. In dieser zusammengewachsenen, klein gewordenen Welt ist es kaum mehr möglich, sich in seinen landeseigenen Kokon zurückzuziehen und fremde Kulturen $\mathrm{zu}$ ignorieren. Statt dessen erfordert diese neue Situation einen veränderten Blick auf die Welt, der die einzelnen Länder, Kontinente und Nationen nicht in Ethnen und Religionen einteilt und klassifiziert, sondern die Welt als Gemisch von Kulturen betrachtet, bei denen das Verbindende hauptsächlich 'der Mensch' sein sollte.

1 Dieser Aufsatz ist die Erweiterung und Überarbeitung eines Vortrags, der von mir am 30. August 2005 in Paris im 11. Kongress der IVG (Internationaler Verein fur Germanisten) gehalten wurde.

Rafik Schami: Mit Vergnügen auf dem Hochseil. Die Arabische Welt. S. 6. Aus: http://www.buchszene.de/neu/arabien/06 07b.pdf. (Kurztitel: Schami: Mit Vergnügen auf dem Hochseil.) 


\section{Dalia Salama}

Eine wichtige Rolle bei dem Dialog der Kulturen spielt die Sprache. Wird die 'andere' Sprache beherrscht, so ist eines der vielen Hindernisse für näheres Kennenlernen abgebaut und der Weg geebnet für den Dialog. Ein Beispiel dafür gibt uns der bekannte syrische Schriftsteller Rafik Schami, der neben Youssef Naoum, Adel Karashouli und anderen zu den sogenannten Migrantenschriftstellern mit arabischem Hintergrund zählt.

Rafik Schami, der schon seit längerer Zeit zu den erfolgreichsten und weltweit meistgelesenen, deutschsprachigen Schriftstellern der Gegenwart gehört, und dessen Bücher bereits in 23 Sprachen übersetzt wurden, spielt eine wichtige und entscheidende Rolle in der Entwicklung der Migrantenliteratur, die seit dem Beginn der Gastarbeiteranwerbung in den fünfziger Jahren einen immer deutlicheren Platz innerhalb der deutschen Literatur eingenommen hat und durch die Vielzahl ausländischer Autoren aus allen Teilen Europas, sowie den arabischen Ländern, und deren Veröffentlichungen in Deutschland über mehrere Jahrzehnte hinweg nun einen nicht wegzudenkenden Teil der deutschen Gegenwartsliteratur darstellt.

Im Juni 2003 verlieh Prof. Dr. E. Jürgen Zöllner, Minister für Wissenschaft, Weiterbildung, Forschung und Kultur, dem syrischen Autor den Kunstpreis $^{3}$ des Landes Rheinland-Pfalz, und würdigte bei einer Festveranstaltung ${ }^{4}$ im Orchestersaal des Staatstheaters Mainz den inzwischen 59-jährigen als „,meisterhaften Erzählkünstler“, der sich in Deutschland nach und nach eine eigene Welt geschaffen - „eine Welt aus Büchern, aber auch eine Welt aus Leserinnen und Lesern". Hohe Auflagen seiner Werke und Lesungen in vollen Sälen seien ein Beleg für das Interesse an diesem Autor, der sich ,in der Sprache seiner neuen Heimat, eine neue Existenz, eine literarische Identität“" erschaffen habe. Für die Jury des Kunstpreises, so Zöllner, die unter seinem Vorsitz getagt habe, ist nicht nur der polykulturelle Hintergrund Schamis ausschlaggebend gewesen. Einziger Maßstab für die Wahl Schamis, der seit 1971 in Deutschland lebt, sei die Qualität seines literarischen Werks. So beschreibt der Minister lobend:

3 Ein Preis, der seit 1956 als höchste Auszeichnung des Landes für Kulturschaffende, jährlich abwechselnd, herausragende Leistungen in der Bildenden Kunst, der Literatur, der Musik und der darstellenden Kunst auszeichnet.

$4 \quad$ Jürgen Zöllner: laudatio aus: Aktuelle Pressemeldungen : Rheinland-pfälzischer Kunstpreis 2003. www.mwwfk.rip.de/Aktuelles/presse2000.asp. (Kurztitel: laudatio) 


\section{Rafik Schami}

Rafik Schami hat die Farben und die Gerüche, die Landschaften, die Städte, die Menschen und ihre Schicksale aus seiner alten orientalischen Heimat mitgebracht als literarische Grundlage seiner Erzählungen. Und mit ihnen zusammen hat er auch die Formen literarischen Erzählens herüber gerettet in eine Sprache und ihre Literatur, die dies nur aus den Märchen aus Tausendundeiner Nacht kennt und liebt. ${ }^{5}$

Zöllner betonte dabei auch, dass jüngere Autorinnen und Autoren mit multikulturellem Hintergrund von Schamis Popularität und Engagement profitierten; er gehöre nämlich zu denen, die „Gastarbeiterliteratur" salonfähig gemacht und diese Klassifizierung zugleich überwunden hätten. Anerkennend bemerkt der Minister:

[...] daß die Veröffentlichungen der jungen Generation nicht mehr in die Schublade Migrantenliteratur gesteckt werden, ist auch das Verdienst dieser Entwicklung, die Rafik Schami maßgeblich mit angestoßen hat. ${ }^{6}$

Bemerkenswerterweise lernte Rafik Schami, dessen ursprünglicher Name Suheil Fadél ist, schon früh, was es heißt, Mitglied einer Minderheit zu sein. Aufgewachsen im arabisch dominierten Damaskus 'lebte er', wie er mit eigenen Worten sagt:

In einer Welt verschiedener Konfessionen und Sprachen. Ich gehörte der christlichen Minderheit in Syrien an, und in dieser Minderheit gehörte ich zur Minderheit der Aramäer. Das waren die Pole meiner Welt.?

Das Pseudonym Rafik Schami, was so viel wie Freund oder Gefährte aus Damaskus bedeutet, ist ein Künstlername, den sich der Autor kreierte, da er noch während seiner Schulzeit hauptsächlich Minderheitenprobleme thematisierte und Kritik an der Unterdrückung durch die Herrschenden ausübte. Als jedoch die kritisch literarische Wandzeitung Al Muntalak (Der Ausgangspunkt), die Schami als Herausgeber und Autor seit 1966 leitete, verboten wurde, emigrierte er nach Deutschland, wo er noch während seines Studiums in Heidelberg wieder begann, Geschichten zu schreiben.

\footnotetext{
5

Laudatio. A. a. O.

6 Ebenda.

7 Rafik Schami: Damals dort und heute hier. Über Fremdsein. Hrsg. Von Erich Jooß. Freiburg; Basel; Wien 1998. S. 16. (Kurztitel: Schami: Damals)
} 


\section{Dalia Salama}

Nach umständlichen und langwierigen Übersetzungen aus dem Arabischen ins Deutsche findet Schami im Laufe der Zeit schließlich in der deutschen Sprache eine neue Heimat und beginnt direkt auf Deutsch zu schreiben. Schami sagt dazu:

Schreiben in einer Fremdsprache ist ein komplexes Geschehen. Es ist eine Gratwanderung zwischen Verzweiflung und paradiesischer Freude, ein Balanceakt auf dem Hochseil mit dem Ziel, den Himmel zu erobern. Ich erfahre täglich bei der Arbeit diese atemberaubenden Augenblicke, während ich arabische Poesie mit der deutschen Sprache vermähle. Das ist schwierig und spannend zugleich. ${ }^{8}$

Schami diente die deutsche Sprache dabei einerseits als Brücke zum deutschen Publikum, und verhalf ihm andererseits auch zum Kontakt mit Autoren anderer Muttersprachen, ${ }^{9}$ mit denen er zusammenarbeitete. ${ }^{10}$ Seit 1982 lebt er als freiberuflicher Schriftsteller in Deutschland, nachdem er seinen Beruf als Chemiker aufgegeben hatte, um sich ganz seiner literarischen Tätigkeit widmen zu können. Bis 1987 erschienen von ihm in rascher Folge mehrere Sammelbände mit Märchen, Fabeln und phantastischen Geschichten, die mittlerweile in mehreren Auflagen und verschiedenen Ausführungen vorliegen. Zu erwähnen wären z. B. hier die Sammelbände Das Schaf im Wolfspelz (1982), Das letzte Wort der Wanderratte (1984), oder Der erste Ritt durchs Nadelöhr (1985). ${ }^{11}$

Wichtig ist an dieser stelle hervorzuheben, dass Schamis Werke nicht nur als interkulturelle Annäherung durch Kritik und Aufklärung zu sehen sind, sondern auch als Kulturvermittlung. In einem seiner Interviews erklärt er:

8 Schami: Mit Vergnügen auf dem Hochseil. A. a. O. S. 7

9 Wie Franco Biondi, Jusuf Naoum oder Suleman Taufik, mit denen er Anfang der 80er die Literaturgruppe Südwind und den polynationalen Literatur- und Kunstverein Polikunst, grundete deren Zielsetzungen darin bestand, sich um die Etablierung der von immigrierten Autoren verfassten Literatur zu bemühen.

10 Vgl.: Lutz Tantow: Abenteuer in der anderen Sprache. In: Die Brücke Nr. 24. April/Mai 1985. (S. 37-42). Hier S. 39 (Kurztitel: Tantow. Abenteuer)

"1 Rafik Schami: Das Schaf im Wolfspelz. Märchen und Fabeln. Dortmund 1982. Das letzte Wort der Wanderratte. Märchen, Fabeln und phantastische Geschichten. München 1984. Derselbe: Der erste Ritt durchs Nadelöhr. Noch mehr Märchen, Fabeln und phantastische Geschichten. Kiel 1985. 
Rafik Schami

Trotz ihrem Übermaß an politischen Nachrichten bringen die Medien nichts über den kulturellen Grund, der uns ausmacht, der ein Teil von mir ist. [...] Es geht darum, die Heimat, die wir verlassen haben, hier bekannt zu machen, damit die Annäherung leichter fällt. ${ }^{12}$

Vor allem mit seinen märchenhaften Erzählungen wurde Schami einer breiten Öffentlichkeit nicht nur in Deutschland bekannt. Die Wahl des Genres Märchen für seine Erzählungen begründet Schami mit der kulturellen Verankerung dieser Gattung in der arabischen Kultur und der orientalischen Erzähltradition, die er im Gegensatz zur neuen Tendenz des realistischen Erzählens ${ }^{13}$ sieht. Neben der kulturellen Identität, haben aber auch die Märchen Rafik Schamis, der ein enthusiastischer Zuhörer der Scheherazade war, deren Geschichten damals im ägyptischen Rundfunk gesendet wurden, seine Kindheit stark beeinflusst, weshalb es ihm nahe liegt, sich ihrer als literarische Ausdrucksform zu bedienen. Darüber äußert er sich selbst:

Und deshalb schreibe ich Märchen nach wie vor neben meinen realistischen Arbeiten, als Stück meiner Identität. Das ist ein Stück von mir, das ist ein Stück meiner Kindheit, meiner Erinnerung, in der ich mich wohlfühle. ${ }^{14}$

Anders als die harmonisierenden Märchen bezeichnet Schami seine als die „anderen" Märchen, denen er die Eigenschaften „rebellisch" und „emanzipatorisch“ zuschreibt. Seine Prämissen für das „rebellische“ Märchen sind, dass es „,weder die Moral der Gehorsamkeit noch das Happy end kennt. Prächtig in den Farben, eindeutig im Inhalt ruft das Märchen rücksichtslos und ohne Pathos zum Widerstand auf." 15 Wie Ulrike Reeg herausstellt intendiert Rafik Schami mit seinen Märchen zweierlei:

12 Tantow: Abenteuer. A. a. O. S. 42.

13 Vgl. Ebenda S. 39

14 Tantow: Abenteuer. A. a. O. S. 38

15. Warten ist ein schlechter Rat in einer eilenden Zeit. Über Illusionäres und Revolutionäres der Phantasie II. In: Linkskurve. Kiel 1983. (H3) S. 38-41. Hier S. 38. Vgl. dazu auch El - Hoda Henaoui: Literarische Werke in deutscher Sprache von Schriftstellern arabischer Herkunft. Untersuchungen zur Gegenwartsliteratur der Bundesrepublik Deutschland in den 80er Jahren. Magisterarbeit. Kairo 2001. S. 38f 


\section{Dalia Salama}

einmal, den Rezipienten in die Lage zu versetzen, Realität zu durchschauen, d. h. das spezifische Verhältnis von Minderheit und Mehrheit, von Starken und Schwachen Unterdrückten, und Unterdrückern, und zum anderen, Möglichkeiten zu ihrer Bewältigung zu eröffnen. ${ }^{16}$

Die Leistungen der Gattung Märchen, die auf der Rezeptionsebene den Leser „verführen" besteht nach Schamis Meinung unter anderem darin, dass sie „durch das scheinbar Unverbindliche, zu verbindlichem Zuhören“ verleitet. Dadurch können für den deutschen Rezipienten oft ungewohnte und nicht immer angenehme Themen zugänglich gemacht werden:

Märchen sind imstande, den Hörer oder den Leser zu gewinnen [...] inhaltlich auf ungeheuer schwere gesellschaftliche Probleme, ungewohntes Zusammenleben mit anderen Kulturen, Entrechtung von einer Minderheit usw. zu stoßen. Aber mit List: wir reden erstmal über Rahmen, höre mal gut zu und überleg's dir selbst. Man muß dem Publikum die Möglichkeit zum Nachdenken lassen. Das ist Märchen. ${ }^{17}$

Allgemein beschrieben stellt Rafik Schamis Literatur den Versuch dar, alte Geschichten neu beziehungsweise neue Geschichten in alter Tradition zu erzählen:

Dabei stehen sich Tradition und Moderne, Orient und Okzident, Minderheit und Mehrheit, Unterdrückte und Unterdrücker nicht starr gegenüber, sondern werden als ineinander fließende Elemente dargestellt, die sich prozeßhaft und in Abhängigkeit entwickeln. ${ }^{18}$

Schami verbindet arabische Erzähltradition mit europäischen Volksmärchen. Seine Erfahrung als Migrant, sowie die Distanz sowohl zu seiner früheren Heimat Syrien, als auch zu seiner Wahl-Heimat Deutschland erlauben ihm einen kritischen, ethnologischen Blick. In seinen Werken wagt Schami den Spagat zwischen beiden Kulturen, und zeigt die Fähigkeit, diese sowohl aus

16 Ulrike Reeg: Schreiben in der Fremde. Literatur nationaler Minderheiten in der Bundesrepublik Deutschland. Essen 1988. S.189 (Kurztitel: Reeg: Schreiben)

Tantow: Abenteuer. A. a. O. S. 38

Heidi Rösch: Migrationsliteratur im interkulturellen Kontext. Eine didaktische Studie zur Literatur von Aras Ören, Aysel Özakin, Franco Biondi und Rafik Schami. Frankfurt a. M. 1992. S. 174. 


\section{Rafik Schami}

der Sicht des Fremden, des arabischen Migranten im Okzident, als auch, wie aus seinen jüngeren Werken sichtbar wird, aus der Sicht eines deutschen Reisenden im Orient zu schildern.

Was Schami von anderen arabischen Exilautoren in Deutschland unterscheidet ist unter anderem seine Auffassung von Migrantenliteratur. Er betont immer wieder, dass Literatur mehr sein muss als Dokumentation. Seiner Meinung nach müssen die Migrantenschriftsteller aufhören, nur ihre Probleme im fremden Land wieder und wieder $\mathrm{zu}$ beschreiben und $\mathrm{zu}$ veröffentlichen. Mittlerweile müssten die Exilautoren vielmehr einen Brückenschlag zwischen ihren Heimatländern und Deutschland versuchen und ihre Literatur um neue Erzählwege bereichern. Sie sollten versuchen, „die Grenzen deutscher Dichtung aufzustoßen und neue Horizonte zu erblicken. ${ }^{619}$ $\mathrm{Ob}$ und inwieweit sich eben diese thematischen Verschiebungen in den Werken Schamis feststellen lassen, soll im Folgenden anhand der Untersuchung zweier Werke exemplarisch herausgestellt werden. Dazu stelle ich die Erzählung Als der Meister auftrat ${ }^{20}$ (1984) (erschienen im Erzählband „Das letzte Wort der Wanderratte") dem relativ neueren Roman Reise zwischen Nacht und Morgen ${ }^{21}$ (1995) gegenüber.

Rafik Schamis Erzählungen stehen in der orientalischen Tradition der „Hakawati“", der Märchenerzähler, die ihre Geschichten in Cafés zum Besten geben. Ein altes Stilmittel dieser Erzähltradition, in Europa aus den Erzählungen von Tausend und eine Nacht wohl bekannt, ist die Verschachtelung von einer oder mehrerer Geschichten in eine Rahmenhandlung, so auch bei der Erzählung Als der Meister auftrat.

Die eigentliche Geschichte, ein „Märchen für Erwachsene“ (M 75), wie es Schami selber nennt, ist in eine Rahmenhandlung eingebettet, die die Erzählsituation selbst thematisiert. Schami stellt sich als Erzähler eines Märchens im Rahmen einer Literaturlesung an der Volkshochschule in Hamburg, dar, setzt sich für eine mündliche Lesung ein und beschreibt ihre Vorteile:

19 Schami: Damals. A. a. O. S. 91 Fabeln und phantastische Geschichten. München 1984. S. 80-101. ( Sigle „M“) 


\section{Dalia Salama}

Anders als bei uns im Süden, wo die Leute laut lachen und heulen, wenn sie eine Geschichte hören, sitzen hier die Zuschauer einer Lesung ruhig wie in einer Kirche, und mancher Schriftsteller gefällt sich in der Rolle eines Pfaffen. Aber zwölf Jahre haben mir den Blick für die Reaktion der Zuhörer geschärft und ich kann die aufgesetzte Maske durchbohren und spüren, ob die Worte meines Märchens die Zuhörer schmerzen oder sie erfreuen. (M 75)

Mit dem Hinweis auf die Unterschiede zwischen den Zuhörern beider Kulturen, weist sich Schami selber auch gleich als Südländer aus. Schami beschreibt die Umstände der Lesung, die als Rahmenhandlung während des eigentlichen Märchenvortrags immer wieder die Erzählung durchbricht. So werden z. B. Kommentare der Zuhörer wiedergegeben oder das Publikum beschrieben. Am Ende des Märchens erscheint ein unerwarteter Gast im Zuschauerraum, eine Gestalt aus dem eigentlichen Märchen. Durch diesen Kunstgriff gewinnt die Erzählung an Realität, sie wird sozusagen als Wahrheit dargestellt.

Obwohl das eigentliche Märchen am Anfang mit den Worten "Es war einmal [...]" (M 75 ) beginnt und sich somit in die Tradition des klassischen Märchen einreiht, sind die allegorischen Bezüge auf die Geschichte der Bundesrepublik Deutschland deutlich zu erkennen:

Es war einmal vor nicht allzu langer Zeit ein König. [...] Gierig, wie nun mal Könige sind, war auch der König von Nordland; er war sogar unersättlich. [...] Er führte Kriege, raubte den unterworfenen Völkern ihr Gold, Eisen und Kupfer und wurde immer mächtiger [...]. Da erdachte sich der König eines Tages einen teuflischen Plan: nicht mehr die Güter der Erde ins Nordland zu bringen, sondern einfach das Nordland über die ganze Erde auszudehnen. Er allein und niemand anders sollte die Erde beherrschen. [...] Der furchtbare Krieg dauerte lange, und das Nordland dehnte sich wider Erwarten nicht aus, sondern schrumpfte immer mehr zusammen bis auf eine Ebene und mehrere kleine Burgen. (M 75f)

Angespielt wird auf die kolonialistische Politik Hitlers, den 2. Weltkrieg und die damit verbundene Zerstörung Deutschlands, sowie die Nachkriegsära. So erinnert die Situation des Nordreichs nach dem verlorenen Krieg deutlich an die Krisenlage Deutschlands nach dem 2. Weltkrieg:

Er [der König] schaute sich verzweifelt um: überall nur Trümmer, und seine Untertanen wühlten im Schutt nach Eßbarem. (M 82) 
Rafik. Schami

In dieser Situation erscheint der Dämon der „Macht und des Reichtums, Akirema“ (M 76), der den König wirtschaftlich unterstützt:

Er verhalf dem König zu Gold und Kupfer, Eisen, Weizen und Mais, und der König versprach dem Dämon ewige Treue. (M 76)

Bei näherem Hinsehen erweist sich der mächtige Dämon Akirema als ein Anagramm von Amerika, die als finanzkräftige Großmacht, Deutschland nach der Niederlage im 2. Weltkrieg wesentlich unterstützte. (Dadurch wird die Übertragung des Märchens in diese Zeit noch einmal verdeutlicht.) 1955 beginnt Deutschland durch Anwerbeabkommen, zunächst mit Italien, die für die Phase des Wiederaufbaus und des wirtschaftlichen Aufschwungs benötigten Arbeitskräfte aus den Mittelmeerländern nach Deutschland zu holen:

Die holen wir uns von meinem Bruder, dem Spaghettikönig, [...] Von meinem Bruder, dem König des Knoblauchreiches, holen wir uns einige seiner Knoblauchfresser, [...] Von meinem geliebten Bruder, dem König der Wüsten, holen wir einige seiner Kameltreiber [...] Der König zählte lange und überzeugend seine Brüder auf $[\ldots]$ (M 77)

In der Weiterführung der Geschichte wird die Situation der Migranten veranschaulicht. Zwei Burgen, die der „Nordländer" (M 78) und die der „Südländer“ (M 78), entstehen, sind jedoch durch einen „gähnenden Abgrund“ (M 78) voneinander getrennt. Die Ausgliederung der ausländischen Arbeitskräfte aus der Gesellschaft kann trotz der gemeinsamen Arbeit nicht verhindert werden:

Unten auf der weiten Ebene waren Felder und Fabriken, wo Nord- wie Südländer gemeinsam schufteten. Nach getaner Arbeit zogen sie sich aber in ihre Burgen zurück. (M 78)

Die Vorurteile, die in den Köpfen der Menschen auf beiden Seiten des Abgrunds herrschen, werden durch den König der Nordländer und seinen Priestern gezielt verstärkt. Treffend werden sie vom Erzähler, der beide Kulturen kennt, beschrieben. Knoblauchfresser, Spaghettis, Kameltreiber, Kümmeltürken oder Kanaken, stehen Hans Wurst, Feldwebel, Sauerkraut und Bierbauch gegenüber. 


\section{Dalia Salama}

Um jegliche Kontaktaufnahme zwischen Nord- und Südländern zu verhindern und immer Distanz zu wahren, werden Manipulierungsmethoden angewandt:

$\mathrm{Ab}$ und $\mathrm{zu}$ hing in der nordländischen Burg ein Plakat mit dem Bild eines leichtsinnigen Nordländers, der den Südländern zu nahe gekommen war. Das Bild zeigte ein krummes Messer, das in den Rippen des Mannes steckte, und obwohl die Leiche zu lächeln schien, erschraken viele Nordländer vor dem Anblick der furchtbaren Waffe. (M 78)

Deutliche Kritik wird an den Medien ausgeübt. Statt zu versuchen diese Vorurteile zu beseitigen, tragen die Medien nur noch zu ihrer Verstärkung bei. So rekurriert die Aufhängung dieses Plakats nicht nur auf die Manipulationsversuche von Medien und Regierungsvertretern, sondern auch auf die Qualität der geschürten Vorurteile. ${ }^{22}$ Indem sie die Gewalttätigkeit der Südländer hervorheben, verstärken sie den Eindruck, dass Zwistigkeiten von den Gastarbeiten schnell mit den Messern beigelegt werden, flößen so Nordländern noch größere Angst ein und verstärken Hassgefühle auf Südländer.

Schami thematisiert hier den Nord-Süd-Konflikt, indem er die Abgründe zwischen den Bevölkerungsgruppen deutlich macht und zeigt, wie bedrohlich sie den Menschen, vor allem den Südländern, werden können. Aufgezeigt wird, wie diese Abgründe verhindern, dass Nord- und Südländer, Deutsche und Migranten, einander näher kommen. Auch die gemeinsame Arbeit der Nord- und Südländer kann nichts daran ändern.

Die mangelhaften Informationen und Kenntnisse der jeweils Anderen führen zur Ausbildung verschiedenster diskriminierender Benennungen und Klischeevorstellungen ${ }^{23}$ :

Die Nordländer $[\ldots]$ hielten die Südländer für eine besondere Art Maschine, die zufällig wie ein Mensch aussieht. (M 79)

Erwähnenswert an dieser Stelle ist, dass die Charakteristika der Deutschen und Ausländer überspitzt hervorgehoben wird. Auf das Leben der Südländer, bei denen die Arbeit den Hauptteil ihres Lebens in Anspruch 


\section{Rafik Schami}

nimmt, wird im Märchen durch das Schild „Die Arbeitsamen“ (M 78), das über dem Gang zu ihrer Burg angebracht ist, hingewiesen. Über dem Gang der Nordländer, die finanziell besser gestellt und wahrscheinlich insgesamt glücklicher sind, da sie weder an Heimweh leiden, noch mit einer neuen und ungewohnten Situation in einem fremden Land konfrontiert sind, prangt das Schild „die Glücklichen“. (M 79 ) Zurückgezogen und abgeschirmt von der Gesellschaft leben die Südländer auf ihrer Burg, und versuchen, so weit es ihnen möglich ist, mit der ihnen aufgezwängten Situation fertigzuwerden, indem sie näher zusammenrücken. Aber auch die Verklärung der alten Heimat durch die Südländer kommt deutlich zum Ausdruck:

Nacht für Nacht saßen die ermüdeten Südländer zusammen, sangen Lieder, erzählten sich die tollsten Geschichten von ihrer Heimat und überboten sich an Übertreibungen. (M 79)

In den Erinnerungen der Südländer wird die alte Heimat idealisiert, und einige von ihnen möchten auch gerne wieder dorthin zurückkehren. So auch Hassan, „ein Südländer, ein Araber“ (M 79), der Protagonist in diesem Märchen:

Hassan war ein lustiger, aber ängstlicher Typ. Er lebte allein in einem ärmlichen Zimmer, denn er wollte eines Tages zurückgehen und einen kleinen Laden in seinem Dorf aufmachen. (M 79)

Doch die Rückkehr in die alte Heimat erscheint noch schwieriger als die Überwindung des Konflikts zwischen Nord- und Südländern, denn „in Richtung Süden klaffte ein noch größerer Abgrund, der die Burg von der südlichen Heimat trennte. "(M 82) Als sich die Möglichkeit eröffnet durch die von den Kindern aus Ästen geschlagenen Brücke den Abgrund zu überqueren und sich immer mehr Verkehr zwischen Nord und Süd zu bilden beginnt, wird dieser jedoch auf Befehl des Königs unterbrochen:

Nicht auf einen Schlag solltet $\mathrm{Thr}$ die südländischen Sklaven zurückschicken wie euer Neffe, der König von Nigeria. Nein, das kann böses Blut geben. Lautlos und Permanent heißt die Devise. Ein stetiges Abschieben höhlt die verdammte Burg aus. (M 82)

Die Politik der Abschiebung, der vor allem in den Zeiten der wirtschaftlichen Rezession angestellte Versuch, die Gastarbeiter zur Rückkehr zu bewegen oder zumindest den Zustrom der Migranten zu stoppen, spiegelt sich im Bild 
des Königs, der seinen Wächtern befiehlt, die Südländer aus seinem Herrschaftsbereich herauszuprügeln. ${ }^{24}$ Erbarmungslos werden die Südländer vertrieben, wobei viele von ihnen in den Abgrund stürzen und ums Leben kommen.

Nicht nur im Märchen, auch in der Realität findet sich die Problematik des Identitätsverlusts: Entweder können die Migranten aus politischen Gründen nicht zurückkehren, oder sie erleben nach ihrer Rückkehr, dass sie auch in ihrer Heimat nicht mehr akzeptiert werden. So fühlen sie sich als Fremde, verloren zwischen zwei Kulturen, ohne genaue Identität.

Die Problematik des Identitätsverlusts, im Text besonders hervorgehoben, wird am Protagonisten Hassan dargestellt. Hassan gibt sich der Illusion hin, als Südländer durch völlige Angleichung an die Nordländer glücklich werden zu können. Bei diesem Assimilationsprozeß, in dem Hassan eine letzte Chance sieht, soll Gnussapna, ein Hexenmeister, helfen. Bei ihm möchte Hassan eine neue Haut erwerben, um zum einen von den Nordländern akzeptiert, zum anderen aber auch vor der Abschiebung in seine Heimat bewahrt zu werden: Der Name des Meisters Gnussapna, der das bewerkstelligen soll, entpuppt sich bei genauer Betrachtung auch als das Anagramm des Wortes 'Anpassung'.

Die Assimilationsaufforderung, mit denen die Ausländer zu einer Aufgabe ihrer kulturellen Identität veranlaßt werden, erfahren in der Erzählung eine Verabsolutierung: sie werden dargestellt als eine grundsätzliche Voraussetzung für ein Leben im Nordland. ${ }^{25}$

Der Weg der Assimilation geht nur langsam vonstatten und ist für Hassan ein langwieriger Prozess und sehr schmerzhaft. So wird ihm z. B. sein neuer Name „Siegfried“ (M 85) sowie die akzentfreie Aussprache der neuen Sprache im wahrsten Sinne des Wortes mit Peitschenhieben eingeprügelt. , [...] nach dem dreißigsten Hieb klappte es wunderbar." (M 86) Seine gemäßigte Begeisterung, als er sich als hellhäutiger, blonder, blauäugiger Siegfried, im Spiegel gegenübersteht und eingehend beobachtet, veranlasst Gnussapna zu folgendem Lob:

\footnotetext{
$24 \quad$ Vgl. ebenda. S. 199.

25 Vgl. ebenda.
} 


\section{Rafik Schami}

Nun sind Sie ein Nordländer geworden. Ein Hassan hätte entweder leichtsinnig "extra prima“ gerufen oder wäre zusammengebrochen und hätte entsetzt gejammert: Scheiße, nix gut. Aber was sagen Sie, Herr Siegfried? Nicht schlecht. Das ist es, was die neue Haut Ihnen gab. Einen Hauch' gesunder Skepsis und einen unschätzbaren, feinen Sinn für das. Mittelmaß. (M 87)

Hassan hat nicht nur äußerlich eine neue Haut bekommen, sondern auch seine gefühlsbetonte, leidenschaftliche Verhaltensweise und Mentalität hat sich geändert. Die ganze Verwandlungsprozedur, der sich Hassan unterziehen musste, um Siegfried zu werden, erfolgte in einer Art Geburt:

Siegfried ging weiter, aber der Korridor wurde immer niedriger, und er mußte sich erst bücken, dann auf allen vieren kriechen. Nach einer Weile war der Gang nur noch ein enges Rohr. Siegfried kroch weiter. Er war erschöpft, aber ein kleines Licht am Ende des Rohres gab ihm Mut und Kraft. (M 86f)

Der Name Siegfried scheint hier bewusst gewählt worden zu sein. Ähnlich wie Siegfried, der Held der Nibelungen, der im Drachenblut badet und damit eine unverwundbare Haut erhält, gewinnt auch hier Hassan eine neue Haut, die es ihm ermöglicht glücklich unter den Nordländern zu leben.

Oberflächlich gesehen scheint er nun auch ein glückliches Leben zu führen. Doch Hassan ist nicht wirklich tot, innerlich ist er noch tief in der Tradition seiner alten Heimat verwurzelt. Die Assimilation fand nur nach Außen hin statt. Ebenso wie Siegfried der Nibelungen besitzt auch dieser neue Siegfried eine verwundbare Stelle. Als er in einer Kneipe ein altes arabisches Lied hört, kommt es zum Eklat:

Die Stimme dröhnte Siegfried in den Ohren. Er versuchte, mit den Gedanken davor zu flüchten, doch die Erinnerung an seine arme Kindheit, an seine harte Arbeit auf den Feldern zog ihn in den Bann des Liedes. Ihm wurde immer heißer in der Seele, und er hielt sich mit beiden Händen die Ohren zu, aber die Worte und die Klänge des Bauernliedes drangen tief in sein Herz. Viele Stimmen stiegen in ihm auf und wurden immer lauter. Plötzlich hörte er Risse. Überall platzte seine Haut mit einem häßlichen Laut auf und zerriß. [...] Knoblauchgeruch stieg ihm in die Nase [...] (M 91f)

Seine neue Identität, die helle Haut, wird in dem Moment zerstört, als das Heimweh, das Sehnen nach seinen eigentlichen kulturellen Wurzeln seiner übermächtig wird. Vom Heimweh geplagt stürzt er sich verzweifelt in den Abgrund. 
Am Beispiel Hassans wird aufgezeigt, dass der Verlust der Identität, der Verzicht auf die Andersartigkeit, zum Scheitern verurteilt ist. Hassan zerbricht unter der Last der Erinnerung und der Sehnsucht nach seinem Heimatland:

Der Umstand, daß die neue Haut dem Heimweh nicht standhält, ist ein dringlicher Appell den Anpassungsforderungen einer Gesellschaft nicht nachzugeben, die die Aufgabe der Identität implizieren. ${ }^{26}$

Assimilation bedeutet für Schami ein totales Aufgeben der eigenen Identität. Statt der Selbstaufgabe schlägt er hier andere Möglichkeiten vor, die zu einem Zusammenleben verschiedener Volksgruppen führen können. In seinem Märchen steht symbolisch dafür das „Brückenschlagen“. Die Brücken führen über den Abgrund, machen Begegnungen und Annäherungen möglich und können dadurch Vorurteile auf beiden Seiten des Abgrundes aus dem Weg räumen:

Die Eltern wollen es nicht glauben, daß ein Südländer genauso lacht und weint wie ein Nordländer, und das er etwas anderes tut, als den ganzen Tag lang Spaghetti essen und sich mit Knoblauch abreiben. Und die Südländischen Eltern lauschten ungläubig den Erzählungen ihrer Kinder, daß ein Bierbauch auch noch einen Kopf hat, der weint und lacht. (...) Immer mehr Nord- und Südländer entdeckten, daß ihre Namen viel bunter und schöner waren als Knoblauch- und Sauerkrautfresser. (M 81)

Bezeichnend dabei ist, dass der Anfang der Begegnungen von Kindern gemacht wird. Es sind die Kinder, die den mutigen Schritt der Grenzüberwindung wagen, vor allem darum, weil bei ihnen die Vorurteile und Klischees noch nicht so tief verwurzelt sind, kindliche Neugier und natürliche Naivität überwiegen. Beispielhaft erscheint auch, dass drei Kinder unterschiedlicher Herkunft, ${ }^{27}$ Mehmet, Costas und Giancarlo gemeinsam den ersten Schritt wagen. Gemeinsam trauen sie sich den Abgrund zu überqueren und auf die Nordländerkinder zuzugehen. Unbeeinflußt von der Welt der Erwachsenen, auf ihrem Willen zur Freundschaft beharrend, sind es die Kinder, die bis heute nicht den Versuch aufgegeben haben, Brücken ans andere Ufer zu schlagen:

26

Ebenda S. 199

An den Namen der drei Kinder verdeutlicht: Mehmet Türkischer Name, Costas, griechischer Name, Giancarlo, italienischer Name. Diese Bevölkerungsgruppen stellen wohl auch den größten Ausländeranteil in Deutschland dar 
Rafik Schami

Hassan starb letzte Woche, aber die Kinder stehen am Abgrund und werfen Zweige. Nordländische und südländische Kinder, es sind immer noch wenige. Der Meister rennt von Ort zu Ort und versucht, den Südländern eine Haut anzudrehen. (M 92)

Noch während des Erzählens findet in der Rahmenhandlung, der titelgebende Zwischenfall statt: Meister Gnussapna persönlich betritt den Leseraum der Volkshochschule und setzt sich zu den Zuhörern. Von den Anwesenden, inklusive des Volkshochschulleiters, wird der Aufruf des Erzählers, seine Geschichte zu glauben, da sie sich von der Anwesenheit des Meisters in der letzten Reihe persönlich überzeugen können, als Trick, als künstlerisches, auf einen besonderen Effekt bedachtes Bravourstück missverstanden:

Der Volksschulleiter trat aus der ersten Reihe auf mich zu. [...] Das Märchen war sehr traurig, aber der Schluß war gekonnt. [...] Gut gemacht, lobte er mich. Was heißt hier, gut gemacht, der Meister sitzt doch hinter ihnen. Der Volksschulleiter lachte und klopfte mir auf die Schulter. Er kündigte den Auftritt einer südländischen Tanzgruppe an. Ich ging kraftlos durch den Saal, gerade als Meister Gnussapna, einen der Ausländer begleitend, den Raum verließ. (M 92 f)

Mit dieser Erzählung wollte Schami den Deutschen einen Spiegel vorhalten. Die deutschen Behörden, die den Migranten anfangs das Leben erschweren, werden genauso kritisiert wie die Integrationsvorstellungen der Deutschen, die von der Minderheit der Gastarbeiter Anpassung an ihren Lebensstil erwarten.

Themen wie diese in Rafik Schamis Geschichten rechtfertigen, dass seine Literatur auch im engeren Sinne zur so genannten "Gastarbeiterliteratur" gezählt werden kann. Rafik Schami und Franco Biondi erklären in ihrem Essay „Literatur der Betroffenheit“ die Ironie, die hinter dem Begriff der „Gastarbeiterliteratur'“ steckt:

Wir gebrauchen bewußt den uns auferlegten Begriff „Gastarbeiterliteratur", um die Ironie, die darin steckt, bloßzustellen. Die Ideologen haben es fertiggebracht, die Begriffe Gast und Arbeiter zusammenzuquetschen, obwohl es noch nie Gäste gab, die gearbeitet haben. Die Vorläufigkeit, die durch das Wort Gast zum Ausdruck gebracht werden soll, zerbrach an der Realität; Gastarbeiter sind faktisch ein fester Bestandteil der bundesrepublikanischen Bevölkerung. ${ }^{28}$ 


\section{Dalia Salama}

„Literatur der Betroffenheit“, der Begriff, für den Schami und Franco Biondi zu diesem Zeitpunkt plädieren, meint Literatur der Einwanderer, also Literatur derer, die sich mit dem Alltag in Deutschland auseinandersetzen und dessen Probleme lösen müssen:

Die Betroffenheit der Autoren [...], die selbst in der Situation stehen oder sich mit der Situation einlassen, teilt sich mit, macht betroffen, auch da und vielleicht gerade da, wo, „ganz einfach“" autobiographische Berichte aus dem Leben der Betroffenen gegeben werden, oft ohne Kenntnis der literarischen Traditionen. ${ }^{629}$

Doch einer solchen thematischen und lokalen Einengung hatte sich Rafik Schami bereits ziemlich früh entzogen. Besonders der Roman Reise zwischen Nacht und Morgen, der im Folgenden vorgestellt werden soll, liefert hierfür ein gutes Beispiel.

Zwischen der Geschichte Als der Meister auftrat (1984) und der märchenhaften Romanerzählung Reise zwischen Nacht und Morgen (1995) liegen elf Jahre, in denen Schami als Märchenerzähler durch Deutschland reiste. Angeregt durch ständig wachsende, verschiedenartige Erfahrungen wechselt auch das Thema, mit dem sich Rafik Schami in diesem Roman beschäftigt. Schami blickt mit einem durch Reise und Erfahrungen gereiften Blick nicht nur auf die Probleme der Migranten in Deutschland, sondern erweitert seinen Horizont. Anstelle einer einseitigen Perspektive, beginnt Schami das Problem zwischen Migranten und Deutschen als beidseitiges zu erkennen und versucht in einer neuen Schreibart sich beiden sowohl $\mathrm{zu}$ nähern, als auch diese einander näher zu bringen. Diese Schreibtendenz hatte sich bereits in seinen Schriften und Reden angedeutet. Anstatt sich über 'Einsamkeit', 'Sprachlosigkeit', 'Identitätsverlust' usw. zu beklagen, fordert Schami nämlich die Suche nach neuen Themen und Formen:

Das ist schon verbraucht, es wurde genug geschrieben über die Einsamkeit, über die Sprachlosigkeit. Man muß nach neuen Wegen suchen [...], wir sind verpflichtet, diese Literatur zu Blüten zu treiben, hervorragende Kunst zu entwickeln, und nicht immer in Reportage- und Mitleidform zu schreiben. [...] Wir brauchen neue Formen, gestützt auf die Wurzeln der eigenen Literatur. ${ }^{30}$

Irmgard Ackermann: Gastarbeiterliteratur als Herausforderung. In: ZS Frankfurter Hefte. (1) 1983. S. 59.

Claus Wenderott [Hrsg.]: Der Orient wohnt in meinem Wort. Geschichten von Rafik Schami für deutsche und ausländische Schüler. Universität Essen. Arbeitsstelle für 


\section{Rafik Schami}

Bereits der Titel des Romans kündigt diese wechselnde Perspektive in der Reise zwischen zeitlichen und im übertragenen Sinne auch räumlichen Topoi an. Erst die Reise, d.h. der Wechsel des Standpunktes, ermöglicht den differenzierten Blick und ein besseres Kennenlernen von Ost und West, von Morgenland und Abendland. Mit Deutschland als gewähltem Heimatland und syrischen Wurzeln weiß Rafik Schami zwischen beiden Polen zu vermitteln. Diese mittlere und vermittelnde Position zwischen dem Orient und dem Okzident kommt schließlich in seinem Roman Reise zwischen Nacht und Morgen zum Ausdruck.

Die Begegnung von Orient und Okzident steht hier im Mittelpunkt. Thematisiert wird der Dialog zwischen unterschiedlichen Kulturen und der Wunsch nach Freundschaft über Landesgrenzen hinweg. Schami plädiert hier für eine ,polykulturelle Gesellschaft, in der an Stelle eines nationalen Chauvinismus eine gegenseitige Bereicherung der Kulturen stattfindet. ${ }^{631}$

Ein Modell der kulturellen Begegnung und Bereicherung wird hier durch den deutschen Circus Samani symbolisiert, der sich auf eine Reise in den Orient begibt und trotz Sprachbarrieren im fremden Land den Menschen die andere Kultur näher zu bringen versucht. Interessant ist hier der arabisch anmutende Name des Circus'. Samani bedeutet nämlich in arabischer Sprache 'meine jetzige Zeit'. Es ist von daher anzunehmen, dass die geschilderte Situation auf die aktuelle, bzw. zeitgenössische Zeit bezogen werden kann. Neben diesem lexikalischen Hinweis, fügt Schami einen weiteren optischen hinzu, wenn er erklärt, dass er das Wort Circus mit C schreibt. Das C, das so rund wie die Manege ist, hat es ihm angetan, denn „da arbeiten meist zehn und mehr Nationen zusammen". 32

Wie in vielen anderen Werken Schamis wird auch hier das Problem des Zusammenlebens von Minderheit und Mehrheit umkreist. So ist die kleine Gemeinschaft der Artisten im Roman ebenfalls polykultureller Natur. Italiener, Deutsche, Araber und Marokkaner leben zusammen und bilden

Migrantenliteratur. Essen 1992. S. 26. (Kurztitel: Wenderott. Der Orient wohnt in meinem Wort.)

31 Heinz Friedrich: Chamissos Enkel. München 1986. (S. 71-76). Hier S. 74.

32 Angelika Ohland: Geschichten sind wie Freunde. Mit märchenhaften Erzählungen aus Damaskus verführt Rafik Schami zu einem offenen Dialog zwischen Orient und Okzident. In: www.sonntagsblatt.de/1996/6/6-18.htm. (Kurztitel: Ohland: Sonntagsblatt) 


\section{Dalia Salama}

sozusagen eine große Familie, also einen Kreis, in dem alle trotz jeweiliger Andersartigkeit gleichberechtigt nebeneinander leben und einander tolerieren. Gleichzeitig ist aber auch gerade diese bunte Vielfalt der Menschen und die individuelle Besonderheit eines jeden einzelnen, ausschlaggebend für eine erfolgreiche, interessante, bereichernde, und sensationelle Circusvorstellung.

Doch obwohl der Circus eigentlich Freude und Vergnügen verspricht, sind auch Vorurteile gegenüber den umherziehenden Artisten überall zu finden. Als Beispiel dafür steht der Empfang des Circus in der Stadt Talte:

Valentin hatte gerade einen Schluck Kaffee genommen, als es plötzlich Steine regnete, erst kleine, dann faustgroße. Die Circusleute sprangen erschrocken auf, und einige wurden von den Steinen getroffen. [...] Panik erfaßte die überraschte Mannschaft [...]. Scharif und Mansur auf der einen und die unbekannten Angreifer auf der anderen Seite tauschten ein paar laute Rufe. [...] Der Circus sei nicht nur unmoralisch, sondern auch ein Werkzeug der verhaßten Regierung in Ulania, erklärte der Mann [...].Wir sind Gäste im Land, Fremde, die auf Hilfe und Freundschaft angewiesen sind. Sag ihm, daß wir Arabien und seine Kultur lieben [...] betonte Valentin. [...] Wir lehnen eure Freundschaft $a b$, erwiderte [der Mann] ruhig. (NM 212ff)

Der Circus verkörpert im Roman eine Minderheit, die in allen Ländern der Welt fremd ist und von den Einheimischen nur schwer akzeptiert wird. Obwohl sich die Fremden darum bemühen, von der Bevölkerung anerkannt zu werden, stoßen sie häufig auf Unverständnis und Ablehnung.

In Schamis märchenhafter Romanerzählung ist neben der Utopie einer in sich harmonischen multikulturellen Gesellschaft deutlich der dialogische Austausch zwischen Orient und Okzident thematisiert. „Die ganze Geschichte ist ein Dialog zwischen einem Europäer und einem Araber", sagt Schami über seine Reise zwischen Nacht und Morgen, „der Europäer aber hat einen arabischen Ursprung, und der Araber ist deutsch erzogen. “33 Dieser Dialog zwischen den Personen findet auch im Aufbau des Romans statt, wie Schami erklärt: „Ich habe die Geschichte von dem Orientalen wie Mosaikbilder aufgebaut, während die Geschichte von Valentin zielstrebig verläuft wie die europäische Erzählweise, also auf ein logisches Ende hin. ${ }^{634}$ Auch wird im zweiten Kapitel des Romans auf die uralten Beziehungen zwischen Orient und

33 Ohland: Sonntagsblatt. A. a. O. 
Rafik Schami

Okzident, die schon im Mittelalter ihre tiefen Berührungspunkte hatten, hingewiesen:

Eine Reise in den Orient hätte Valentin gern auch ohne die Millionen unternommen [...] seit dem Tod seiner Frau las er sehnsüchtig die Berichte seiner Vorfahren über ihre Reisen in den Orient. Vom Mittelalter an waren die Samanis, die bis zum Jahre 1900 Ruprecht hießen, Gaukler, und seit den Kreuzzügen hatte die Familie Ruprecht ihre Beziehung zum Orient nicht mehr abreißen lassen. So wollte der Großvater von seinem Großvater gehört haben, daß dieser seinen Großvater auf einer Reise in den Orient begleitet hatte. ( NM 24)

Valentin, jetziger Zirkusdirektor und früher ein begabter und mutiger Seiltänzer; ein Talent, dass er von seiner Mutter geerbt hatte, sucht also seine Wurzeln im Orient, denn im geheimen Tagebuch seiner Mutter machte er die Entdeckung, dass er einen arabischen Vater und zwei arabische Halbschwestern hat. Dialogpartner ist sein arabischer und heißgeliebter Freund Nabil, der einst in Deutschland war und mit dem ihn schon seit seiner Kindheit nicht nur eine enge Freundschaft, sondern auch Blutsbruderschaft verbindet. Auch Nabil empfindet große Liebe und tiefste Verbundenheit zu Valentin, wie es deutlich in seinem Brief erkennbar ist:

Wir waren beide nicht einmal fünfzehn und haben uns befreundet und Treue geschworen. Du hast mir viele Geheimnisse des Circus gezeigt. Ich durfte zu jeder Zeit zu Dir kommen. Und ich habe Dir die Geheimnisse von Ulania gezeigt. Und ob Du es mir glaubst oder nicht, Dir zuliebe habe ich mich als Kind für die Deutsche Schule entschieden. [...] Mein Vater fragte mich, in welcher Schule ich weiterlernen wolle. Ich sagte in Gedanken an Dich: In der deutschen Schule! (NM 20 f)

Nicht nur seine Liebe zu.dem todkranken Nabil und die Errettung aus der finanziellen Misere führen Valentin, der eine Liebesgeschichte schreiben möchte, in den Orient:

War es die Einsamkeit nach dem Tode seiner Frau oder die Sehnsucht nach dem Land seines wirklichen Vaters und seiner Großmutter Alia? Wollte er nur die Spuren der Liebe verfolgen, oder wollte er wissen, wo er herkam? Ob es nun das eine, das andere oder alles zusammen war - seine Sehnsucht nach Ulania war entfacht. [...] vor seinem inneren Auge sah er die Fäden immer wieder im Orient zusammenlaufen. [...] Valentin betrachtete nun den Orient nicht mehr als irgendeine Gegend dieser Welt. Die Sehnsucht nach dem Orient wohnte jetzt in ihm, freilich eine Sehnsucht nach etwas, wovon er nur eine vage Vorstellung besaß. [...] Die Aufzeichnungen seiner Mutter faszinierten ihn, doch sie halfen ihm für die Atmosphäre der 


\section{Dalia Salama}

Geschichte nicht weiter. Die Landschaft, die Menschen und wie sie miteinander lebten - über all das fand sich im Tagebuch der Mutter nichts. (NM 39f)

Durch die finanzielle Unterstützung Nabils, wird es Valentin ermöglicht, sich samt seinem Circus auf die langersehnte Reise in den Orient zu begeben. Schami wählt dabei eine interessante Erzählperspektive für seinen Roman. So wird die bisher übliche Erzählperspektive der Migrantenliteratur umgekehrt: Statt Deutschland aus der Sicht des Fremden zu beschreiben, schildert der deutsche Protagonist Valentin seine Gefühle in der Fremde Arabiens. Zwar hat der deutsche Circusdirektor eineri mabischen Vater und lebte sogar in seiner Kindheit für kurze Zeit im Orient, fühlt sich aber trotzdem zunächst ziemlich fremd. Schami bedient sich einer Technik, die es ihm ermöglicht, den Orient Gegenstand objektiver Reflexion zu machen. Indem er nämlich die arabische Heimat durch die deutschen Augen Valentins und seiner Freundin Pia beschreibt, schafft er sich die nötige Distanz und somit die Fähigkeit zu sachlicher Kritik. Dabei ist besonders interessant, wie unterschiedlich die Fremde auf Valentin und Pia, die Valentin, ihrer Liebe nachgereist ist, wirkt. Während Valentin sich mit Liebe dem Land und seinen Bewohnern öffnet, mit Begeisterung die arabische Sprache erlernt und vorrangig Aspekte der Fremde, das Exotische in seinen Erlebnissen beschreibt, sieht Pia alles kritischer und distanzierter. (NM 322ff) Durch den erzähltechnischen Kniff, gemeinsam Erlebtes sowohl von Valentin, als auch von Pia beschreiben zu lassen, schafft es Schami, dem Leser zu verdeutlichen, dass Erfahrungen in der Fremde immer subjektiv und oft durch Vorurteile und unbewußte Erwartungen an das Land geprägt sind. Diese ungewöhnliche Art der Beschreibung ist Schami nur deshalb möglich, weil er beide Kulturen, sowohl die deutsche als auch die arabische, kennt und sich in beide Seiten hineinversetzen kann. Durch die Beschreibungen des täglichen Lebens, des Basars und des Hammams, der politischen Verhältnisse, sowie das Aufzeigen einfacher Verhaltensregeln und Sitten vermittelt er dem Leser ein facettenreiches Orientbild. und legt ihm eine orientalische Welt vor, die aus der Sicht von Individuen gesehen wird und nicht auf Kollektiv- und Pauschalurteilen basiert.

Auch die orientalische Erzähltechnik der Verschachtelung von Geschichten wendet Schami in diesem Werk wiederum an. Dabei wird das Moment des Erzählens, das die beiden Romanfiguren Valentin und Nabil einander näherbringt, zunutze gezogen. Spät abends, am „Nachmorg“ (NM 117), wie die alten Freunde Nabil und Valentin die Zeit zwischen Nacht und 


\section{Rafik Schami}

Morgen nennen, sitzen sie oft zusammen und erzählen von vergangenen Zeiten, wobei sich in Geschichten über Geschichten Besinnliches, Heiteres und Dramatisches entfaltet. Folgende Textstelle zeigt die Bedeutsamkeit dieser Zeit für Nabil:

Wie spät ist es?" , fragte Valentin. Erschlage die Schönheit dieser Zeit nicht mit der Uhr, antwortete Nabil, es ist die schönste des Lebens, deshalb sterben die meisten Menschen in ihr. Es ist die Zeit, in der die Nacht sich anschickt zu gehen und der Morgen noch nicht ganz angekommen ist. Der Farbe nach Nacht, schmeckt sie bereits nach Morgen. Ich nannte diese Zeit einst Nachmorg, und dieser Name ist geheimnisvoll wie sie. [...] Nebeneinander in bequemen Sesseln sitzend, schworen die beiden Freunde, daß sie sich von nun an immer am Nachmorg aussprechen und ihre Geheimnisse austauschen würden. (NM 117)

Die Aussprache und der Austausch gegenseitiger Geheimnisse am Nachmorg wird zu einem regelmäßigen Ritual, der Nähe und gewisse Intimität in sich birgt und gleich einem Schwur oder Versprechen für die Zukunft über beide Freunde schwebt. Die Bezeichnung „Nachmorg“ ist eine Zusammensetzung von Nacht und Morgen, in der Morgenland und Abendland symbolisch miteinander verschmelzen, und damit auf den Dialog zwischen Orient und Okzident hindeuten. Gleichzeitig weist diese Kontamination aber auch auf eine weit in die Zukunft hinausreichende Zeit hin. Eine Zeit, die nach Morgen kommt, die gleich einem Gelöbnis, Hoffnung in sich birgt.

Genau diese Botschaft vermittelt Schami in einer im Jahre 2003 gehaltenen Rede, mit dem Titel: Dem Morgen begegnen, heißt Hoffnung haben. ${ }^{35}$ In dieser plädiert Schami für den gegenseitigen Austausch zwischen Morgenland und Abendland, der nur durch den Dialog ermöglicht wird und somit die Hoffnung für ein besseres Zusammenleben von verschiedenen Kulturen, die einander bereichern sollen, darstellt. Durch dieses sich Öffnen und Einander näher kommen am „Nachmorg“ geschieht auch in seinem Roman etwas Seltsames und Märchenhaftes, das auf die gegenseitige Bereicherung zurückzuführen ist. Von Mal zu Mal scheinen die beiden alten Freunde jünger zu werden. Auch von der heimtückischen Krankheit Nabils merkt man nichts mehr. Valentin wird selbstbewusster und fasst neuen

Rafik Schami: Dem Morgen begegnen, heißt Hoffnung haben. 7 Bemerkungen eines hoffnungsvollen Pessimisten. Rede auf dem 9. Bundeskongreß für politische Bildung. Braunschweig 6.-8. März 2003. Aus: http://www.bpb.de/veranstaltungen/ ODPLEY 0,0 ,Dem Morgen begegnen hei\%DFt Hoffnung haben.html. (Kurztitel: Schami: Dem Morgen begegnen) 
Lebensmut und findet in Pia eine neue Liebe. Die Liebe von Valentin zu Pia führt bei dem alten Circusdirektor zu einem Verjüngungsprozeß. Pia hingegen beschließt älter zu werden, um ihren Geliebten treffen zu können - sie begegnen sich in der Mitte.

Neben den Circusvorstellungen und den wundersamen und märchenhaften Geschichten, die Valentin und Nabil einander erzählen, heben sich die Konturen der Wirklichkeit in der Reise zwischen Nacht und Morgen deutlich ab. Das fiktive, orientalische Land Ulania stellt ein Phantasieland dar, welches aber nicht gänzlich von der Realität losgelöst ist. Es könnte irgendwo im arabischen Orient zu suchen sein, da es nicht von Konflikten wie Diktatur, Armut und Fundamentalismus verschont wird. All das spiegelt sich auch an verschiedenen Stellen im Fortlauf der Geschichte wieder. So geraten der Zirkusdirektor Valentin Samani und sein alter Freund Nabil, während sie die Bänder ihrer Freundschaft festigen, in die Fänge der Mächtigen und unter das Joch der Fundamentalisten. In einem Interview sagt Schami dazu:

Das ist das politische Thema des Buches [...]sie können weder pro noch contra sein. Eine dritte Haltung, gegen die Mächtigen und gegen die Oppositionellen, gegen die Gewalt also, egal von welcher Seite, findet sich neben den wundersamen Geschichten, die Valentin und Nabil einander am „Nachmorg“ erzählen. ${ }^{36}$

Diese neue Positionierung kann als Richtlinie für den gesamten Roman angesehen werden. Es ist dieses 'zwischen', das bereits im Titel des Werkes angedeutet wird und durch einer 'mittleren' Position und einer daraus resultierenden neuen Perspektive angestrebt wird. In einer Schlüsselszene zeigt Schami, wie diese 'mittlere Position' zu erreichen ist. Das dreitägige Volksfest, das in Ulania stattfindet, und mit dem die Regierung zeigen will, „wie stabil sie war und wie gut Ulania in ihren Händen gedieh" (NM 289) kann als Höhepunkt des Romans gesehen werden. Die „offizielle Feier für die Staatsgäste [...], die im gut erhaltenen griechischen Amphitheater beginnen sollte" (NM 290) wird von der Regierung, ähnlich wie bei den Olympischen Spielen, als Friedensfest zwischen den Völkern vermittelt, in dem alle Kulturen gemeinsam vereint sein sollen: 


\section{Rafik Schami}

Von dort sollten die Teilnehmer etwa fünfhundert Meter weit durch eine Allee zu Fuß zum Stadion gehen. Diesen Weg soliten Marmorsäulen säumen, auf denen Männer und Frauen in historischen Kostümen den Vorbeiziehenden zeigten, in welcher Epoche in der Geschichte Ulanias sie sich gerade befanden. Es sollte eine Art historischer (!) Prozession werden, bei der die Herrschaft der Aramäer, Ägypter, Juden, Griechen, Römer, Araber, Kreuzzügler, Tataren, Türken, Franzosen und zuletzt das dreißigjährige Wechselbad verschiedenster Regierungen dargestellt wurde. Im Stadion wollte der Präsident unter einer bronzenen Reiterin, die Ulania darstellte, ein ewiges Feuer entzünden. (NM 290)

Im Schlussakt der Geburtstagsfeier Ulanias, in dem Eva die Seiltänzerin des Circus Samani ihre Kunststücke auf dem Hochseil vorführt, wird deutlich, dass die Absicht der Regierung, die Leute einander näher zu bringen nur Schein ist, der politischen Interessen dienen soll:

Auf die Sekunde genau stieg Eva zum Hochseil hinauf, das zwischen dem Glockenturm der Kirche der heiligen Maria und dem Minarett der Moschee Saladins gespannt war.[...] Radio und Fernsehen hatten das Ereignis im Laufe der letzten Tage zum eigentlichen Höhepunkt der Feier erklärt und verkauften den artistischen Spaziergang auf dem zwanzig Millimeter dicken, kalten Stahlseil ganz im Sinne der Regierung als Symbol der Verständigung und Verbindung zwischen Islam und Christentum. Doch nicht die Verbindung zwischen Glockenturm und Minarett entsprach der Beziehung zwischen Islam und Christentum, sondern der lebensgefährliche Balanceakt auf dem Seil. Von der Symbolik des Seilgangs unbeeindruckt waren kleine tüchtige Händler vor allem daran interessiert, etwas Geld an diesem spannenden Ereignis zu verdienen. (NM 293f)

Anders als das auf privater Ebene stattfindende persönliche und intime Gespräch unter vier Augen gestaltet sich eine Annäherung auf politischer Ebene als öffentlicher und offizieller Balanceakt, der eine gewisse Akrobatik verlangt, Gefahren in sich birgt und tödlich enden kann. Von den Medien begleitet wird dieses Ereignis bereits zum 'Event'.

In dieser Episode sind mehrere Elemente von symbolischem Gehalt zu erkennen. Só ist der Name der Seiltänzerin, Eva, zugleich auch als Mutter der Menschen zu lesen, die diese trotz ihrer unterschiedlichen Religionen miteinander verbrüdert.

Das Hochseil, auf dem sie ihre Kunststücke vorführt ist zwar zwanzig Millimeter dick, doch kalt und aus Stahl. Diese kalte Verbindung kann als Anspielung auf den kalten Dialog der Politiker und vieler Medien verstanden werden. 


\section{Dalia Salama}

Offensichtlich ist die Bedeutung der Tatsache, dass das Hochseil zwischen dem Glockenturm der Kirche der heiligen Maria und dem Minarett der Moschee Saladins gespannt ist, um eine Verbindung zwischen den Religionen herzustellen. Schami wählt hier bewusst den Namen Saladins, um auf die historische Gestalt des "noblen" Salah-ed-Din El Ayubi hinzuweisen und an die tolerante Politik des damaligen Herrschers zu erinnern. ${ }^{37}$ Dieser wurde nach seiner späteren Einnahme Jerusalems auch von Seiten vieler Europäischer Christen gelobt. Genauso 'wie in der Vergangenheit positive Verhältnisse zwischen Christen und Muslime, Orient und Okzident möglich waren, wird auch für 'heute' bzw. 'morgen' eine ähnliche Beziehung erhofft. Schami meint in seiner Rede hoffnungsvoll dazu:

Viele [...] werden die Übertragung der Hoffnung auf den Orient für eine Utopie halten. Aber es ist gelebte Geschichte. In Spanien hatte diese Synthese von Moslems, Juden und Christen mehrere Jahrhunderte Bestand. ${ }^{38}$

Unübersehbar ist der lebensgefährliche Seilakt Evas und seine Symbolik. Um die Verbindung zwischen Minarett und Glockenturm herzustellen sind nämlich Offenheit, freier Wille, Mut und Bereitschaft zum Wagnis, Begabung und künstlerisches Talent notwendige Voraussetzungen.

„Mit Vergnügen auf dem Hochseil tanzend und von Leichtigkeit berauscht", so formuliert Schami sein Empfinden bei dem freien Vortrag seiner Erzählungen. ${ }^{39}$ Auch die Symbolik des gefährlichen Seilakts findet in Schamis oben erwähnter Rede Ausdruck, als er nach den Ereignissen des 11. Septembers und dem darauf folgenden amerikanischen Kriegsaufbruch in den Irak die ,historische Einmaligkeit“ von Schröders kriegsablehnender Politik, hervorhebt. So meint Schami:

Zum ersten Mal in der Geschichte nach dem zweiten Weltkrieg widerspricht ein deutscher Politiker der amerikanischen Politik, ohne antiamerikanisch zu sein.

37 Vgl. Moustafa Maher: Das Bild des Deutschen in der arabischen und das B Ici des Arabers in der deutschen Literatur. In: Araber und Deutsche. 1978

38 Schami: Dem Morgen begegnen. A. a. O. S. 2 


\section{Rafik Schami}

Achtung muss man Bundeskanzler Schröder für diese Haltung zollen. Es ist ein Tanz auf dem Hochseil ohne Netz. ${ }^{40}$

Durch seinen Mut, 'ohne Netz' den amerikanischen Kriegsaufrufen zu widersprechen, hat Schröder Welten einander näher gebracht. Den Kontakt und die Beziehung zu Amerika zu wahren, ohne dabei als Antiamerikaner zu gelten bedarf in der Tat einer Kunst und ähnelt einem vorbildlichen artistischen Spaziergang auf dem Hochseil. In diesem Sinne sollten sich auch die Menschen verschiedener Nationen bemühen, diesen gefährlichen Seilakt zwischen Islam und Christentum, Orient und Okzident, tanzend und mit Vergnügen $\mathrm{zu}$ vollziehen, anstatt mit Hilfe von gewalttätigen und kriegerischen Mitteln einen Frieden erzwingen zu wollen.

Fazit Schamis ist also Folgendes: die öffentliche Schaustellung eines Dialogs zwischen beiden Kulturen ist oft nur oberflächlicher Natur; ein gewinneinbringendes Event schlechthin. Diesem stellt er ein anderes Modell gegenüber. Nach dem Modell von Nabil und Valentin sind die bequemeren persönlichen Begegnungen und das nähere Kennen lernen von Mensch zu Mensch intensiver und effektiver.

Schami setzt auf die Liebe zwischen den einzelnen Menschen und den einzelnen Personen unabhängig von jeder Politik und politischem Machtgehabe. In diesem Sinne sollten Menschen unabhängig von jeder Politik aufeinander zugehen und sich durch Freundschaft und Liebe nähern. Ein unterschiedlicher kultureller, religiöser und mentaler Hintergrund, kann nur zur Bereicherung in allen Lebenssituationen führen, vitale Lebenskraft spenden und Lebensfreude vermitteln. Schami meint deshalb zum Dialog der Kulturen:

Der Dialog lebt von der Differenz. Die ängstliche Suche nach Harmonie mündet in eine unfruchtbare Gleichmacherei. ${ }^{41}$

Zusammenfassend betrachtet kann man deutlich eine Verschiebung der Thematik bzw. eine Verlagerung des Akzents in Schamis neuerem Werk feststellen. In seiner Erzählung Als der Meister auftrat von 1984 behandelte Schami noch die traditionellen Themen der Migrantenliteratur, wie

Schami: Dem Morgen begegnen. A. a. O. S. 10 


\section{Dalia Salama}

Identitätsverlust oder Heimatproblematik und wirft die Frage auf, inwieweit sich Migranten in ihrer neuen Heimat assimilieren können und müssen. Außerdem macht er Vorschläge, wie das Zusammenleben von Minderheit und Mehrheit gestaltet werden kann, das durch den Brückenbau symbolisiert wird. Dieser sachte Versuch der Grenzüberschreitung, der in dieser Geschichte als Lösungsmöglichkeit angedeutet wird, tritt in seinem neueren Roman Reise zwischen Nacht und Morgen stärker zum Vorschein.

Schami behandelt diese Themenbereiche erneut, jedoch mit unterschiedlicher Akzentuierung und tieferem Blick. Indem Schami den Roman um Themen wie Liebe und Freundschaft zwischen Arabern und Deutschen und polykultureller Gesellschaftsformen erweitert, bietet er Lösungsmöglichkeiten für die bisher in seinen früheren Werken geschilderten Migrationsprobleme. Diesen setzt er nämlich - schon weit vor dem 11. September des Jahres 2001 - den Dialog entgegen.

Zusätzlich ermöglicht die veränderte Perspektive, von der aus Schami seinen Roman verfasst hat, eine kritische Auseinandersetzung mit seinem ursprünglichen Heimatland. Anstelle einer fixierten Perspektivisierung erfolgt nun der duale Blick in beide Richtungen. Dieser erst erlaubt mittels des Dialogs - hier dargestellt im Gespräch zwischen den beiden Freunden Nabil und Valentin - eine Symbiose von scheinbar Gegensätzlichem, nämlich zwischen Orient und Okzident, Morgenland und Abendland, zu einem harmonischen Gefüge, das seinen Höhepunkt im so genannten „Nachmorg“ findet.

Die Aktualität seiner Themen und seine eher ungewöhnlicie Erzählweise in Form von Märchen, die er nicht nur schreibt, sondern auch in ganz Deutschland mündlich erzählt, machen Rafik Schami zu einem Migrantenschriftsteller, dessen Werke nicht nur gedruckt, sondern auch gelesen werden. ${ }^{42}$ Die Wahl des mündlichen Weges ermöglicht Schami die Reaktion der Hörer bzw. des Publikun synchron aufzunehmen und zu registrieren. Zugleich wird ein Gefühl der Nähe und der Wärme vermittelt, das die Ketten der schriftlichen Anonymität sprengt. Dies steht durchaus mit Schamis Anliegen in Einklang, stets die unmittelbare, menschliche Nähe zu suchen, die auf wechselseitiger entpolitisierter Basis beruht. Nach Schami kann also ein kultureller Austausch zwischen verschiedenen Polen nur fernab

t2 Vgl. A. Halstenberg: Einführung zu Rafik Schami liest in. Funkhaus Hannover „Erzähler der Nacht". Aufnahme aus dem Rundfunk. 
von der Politik vertieft und gefördert werden. Darum ist auch für ihn Schröders Haltung der amerikanischen Initiative gegenüber so bemerkenswert, da er es gewagt hat, als Politiker auf menschlicher Ebene zu denken und aus Menschlichkeit zu handeln.

Das Einbringen aktueller Bezüge und das Variieren der Themen in den Werken Schamis machen ihn zu einem Schriftsteller, dessen "so genannte Migrantenliteratur sich schon lange nicht mehr auf die Betroffenheitstexte früherer Jahre beziehen lässt. “43 Brücken der Verständigung zwischen den Kulturen zu schlagen, für Freundschaft und Toleranz gegenüber Fremden und Minderheiten zu werben, ist jedoch bis heute Schamis literarisches Anliegen geblieben.

Zwischen Seilakt und Brückenbau, hin und her bewegend, zwischen Morgenland und Abendland erweist sich Rafik Schami als hartnäckiger Mittler zwischen den Kulturen. Nicht umsonst gilt der erfolgreiche Schriftsteller, der vor über 30 Jahren von Syrien nach Deutschland kam, und der als „Brückenbauer zwischen den Welten" ${ }^{\circ 44}$ angesehen wird, wie ihn die Neue Züricher Zeitung nennt, für viele als Instanz, als einer, der über das schwierige Miteinander von Orient und Okzident zu berichten weiß.

\section{Literaturverzeichnis}

\section{Primärliteratur}

SCHAMI, Rafik: Das Schaf im Wolfspelz. Märchen und Fabeln. Dortmund 1982.

DERSELBE: Als der Meister auftrat. In: Ders. Das letzte Wort der Wanderratte. Märchen, Fabeln und phantastische Geschichten. München 1984. S. 80-101.

DERSELBE: Der erste Ritt durchs Nadelöhr. Noch mehr Märchen, Fabeln und phantastische Geschichten. Kiel 1985.

DERSELBE: Reise zwischen Nacht und Morgen. München; Wien 1995

43 Wenderott: Der Orient wohnt in meinem Wort. A. a. O. S. 25

44 Zürische Zeitung: Zum Thema „Zwischen den Welten“. Sendedatum 21. Juni 2003. Aus: http://www.wdr.de/tv/vetro/archiv/rafik schami.phtml 


\section{Dalia Salama}

DeRSElBe: Mit Vergnügen auf dem Hochseil. Die Arabische Welt. S. 6. Aus: http://www. buchszene.de/neu/arabien/06 07b.pdf.

DERSELBE: Damals dort und heute hier. Über Fremdsein. Hrsg. Von Erich Jooß. Freiburg; Basel; Wien 1998.

DERSELBE: Dem Morgen begegnen, heißt Hoffnung haben. 7 Bemerkungen eines hoffnungsvollen Pessimisten. Rede auf dem 9. Bundeskongreß für politische Bildung. Braunschweig 6.-8. März 2003. Aus: http://www.bpb.de/veranstaltungen/ ODPLEY, 0,0 , Dem Morgen begegnen hei\%DFt Hoffnung haben.html

DERSELBE: Warten ist ein schlechter Rat in einer eilenden Zeit. Über Illusionäres und Revolutionäres der Phantasie II. In: Linkskurve. Kiel 1983. (H 3) S. 38-41.

\section{Sekundärliteratur}

ACKERMANN, Irmgard: Gastarbeiterliteratur als Herausforderung. In: ZS Frankfurter Hefte. (1) 1983.

BANSE, Renate: Deutschsprachige Literatur ausländischer Autoren am Beispiel Rafiq Schami. In: Lernen in Deutschland. Zeitschrift für die pädagogische Arbeit mit ausländischen Kindern und Jugendlichen. H. 2. Baltmannsweiler 1990. S. 52-56́.

El-HeNaOUI, Hoda: Literarische Werke in deutscher Sprache von Schriftstellern arabischer Herkunft. Untersuchungen zur Gegenwartsliteratur der Bundesrepublik Deutschland in den 80er Jahren. Magisterarbeit. Kairo 2001.

FrIEDRICH, Heinz: Chamissos Enkel. München 1986. S. 71-76.

HALSTENberG, A.: Einführung zu Rafik Schami liest im Funkhaus Hannover „Erzähler der Nacht“. Aufnahme aus dem Rundfunk.

HAMM, Horst: „Fremdgegangen-Freigeschrieben“ Würzburg 1988.

HORN, Dieter.: Entwicklungstendenzen in der Ausländerliteratur. In: Sprache und Literatur im Unterricht. (Bd. 9) Baltmannsweiler 1989.

Derselbe: Schreiben aus Betroffenheit - Die Migrantenliteratur in der Bundesrepublik. In: Tumat, Alfred J. [Hrsg.]: Migration und Integration. Ein Reader. Baltmannsweiler 1986. (S. 213-233)

Maher, Moustafa: Das Bild des Deutschen in der arabischen und das Bild des Arabers in der deutschen Literatur. In: Araber und Deutsche. 1978.

OHLAND, Angelika: Geschichten sind wie Freunde. Mit märchenhaften Erzählungen aus Damaskus verführt Rafik Schami zu einem offenen Dialog zwischen Orient und Okzident. In: www. sonntagsblatt.de/1996/6/6-18.htm. 
Rafik Schami

REEG, Ulrike: Schreiben in der Fremde. Literatur nationaler Minderheiten in der Bundesrepublik Deutschland. Essen 1988.

RÖSCH, Heidi: Migrationsliteratur im interkulturellen Kontext. Eine didaktische Studie zur Literatur von Aras Ören, Aysel Özakin, Franco Biondi und Rafik Schami. Frankfurt a. M. 1992.

SCHAFFERNICHT, Christian: Zu Hause in der Fremde. Fischerhude 1981.

Tantow, Lutz: Abenteuer in der anderen Sprache. In: Die Brücke Nr. 24. April/Mai 1985. (S. 37-42)

DERSELBE: Märchen und Satire in der aktuellen „Gastarbeiter“ - Literatur. Zusammenfassung der Arbeitergruppe. In: Evangeiische ihidemie Iserlohn. (Tagungsprotokoll) Ausländer- oder Gastarbeiteriteratur? Geschichte und ikturifle Situation einer neuen Literatur in Deutschland. H. 25. 1985 S. $70-76$.

WEIGEL, Sigrid: Schreibweisen einz'ser Autoren zwischen Kulturen. In: Literatur der FremdeLiteratur in der Fremde. In: Hansers Sozialgeschichte der deutschen Literatur vom 16. Jahrhundert bis zur Gegenwart. Hrsg. Von Rolf Grimminger. Bd. 12 München; Wien 1992. S. 182-229; zu Rafik Schamis Märchen S. $221 \mathrm{f}$

WENDEROTT, Claus (Hrsg.): Der Orient wohnt in meinem Wort. Geschichten von Rafik Schami für deutsche und ausländische Schüler. Universität Essen. Arbeitsstelle für Migrantenliteratur. Essen 1992.

ZÖLLNER, Jürgen: laudatio aus: Aktuelle Pressemeldungen: Rheinland-pfälzischer Kunstpreis 2003. www.mwwtk.rip.de/Aktuelles/ presse2000. asp.

ZÜISCHE Zeitung: Zum Thema „Zwischen den Welten“. Sendedatum 21. Juni 2003. Aus: http://www.wdr.de/tv/vetro/archiv/rafik schami.phtml 